\title{
UNIFORM LIMIT POWER-TYPE FUNCTION SPACES
}

\author{
CHUANYI ZHANG AND WEIGUO LIU
}

Received 10 October 2005; Revised 16 June 2006; Accepted 5 July 2006

To answer a question proposed by Mari in 1996 , we propose $U \mathscr{L} \mathscr{P}_{\alpha}\left(\mathbb{R}^{+}\right)$, the space of uniform limit power functions. We show that $\mathcal{u} \mathscr{L} \mathscr{P}_{\alpha}\left(\mathbb{R}^{+}\right)$has properties similar to that of $\mathscr{A} \mathscr{P}\left(\mathbb{R}^{+}\right)$. We also proposed three other limit power function spaces.

Copyright (c) 2006 Hindawi Publishing Corporation. All rights reserved.

\section{Introduction}

In literature of Fourier transforms and Wavelet transforms, the basic space is $L_{2}(\mathbb{R})$. From the point of view of signal analysis, a signal $f \in L_{2}(\mathbb{R})$ can only be transient (or "wavelets"). During recent years in some application areas, it has become more common to motivate a theory via persistent rather than transient signals (e.g., $[16,28,30,42]$ ). To work on persistent signals, people have to seek a space different from $L_{2}(\mathbb{R})$. One important example of such spaces is $\mathscr{A} \mathscr{P}(\mathbb{R})$, the space of almost periodic functions. People have developed a profound theory and applications for $\mathscr{A} \mathscr{P}(\mathbb{R})$ (e.g., see $[4,5,7-$ $15,18,19,24,26,30,32,37,38])$.

As in [30], a function $f$ is called limit power if the limit

$$
\lim _{T \rightarrow \infty} \frac{1}{2 T} \int_{-T}^{T}|f(t)|^{2} d t
$$

exists. Denote by $\mathrm{H}_{2}$ the set of all such functions.

It is well known that $\mathscr{A} \mathscr{P}(\mathbb{R}) \subset H_{2}$ and so is the Besicovitch space $B_{2}$ [5], the completion of $\mathscr{A} \mathscr{P}(\mathbb{R})$ in $\mathrm{H}_{2}$. In fact, many useful persistent signals are in $H_{2}$, for example, the bounded power signals studied in Wiener's generalized harmonic analysis [36]. However, $\mathrm{H}_{2}$ is not a linear set. An example in [29] shows that $H_{2}$ is not closed under addition. The lack of closedness under addition caused some difficulties in Robust control (e.g., see [27]).

As [29] pointed out that except for some subsets of $H_{2}$ which are already known to be vector spaces (e.g., $L_{2}(\mathbb{R}),\left\{f \in L_{\infty}(\mathbb{R}): \lim _{|t| \rightarrow \infty} f(t)\right.$ exists $\}, \mathscr{A} \mathscr{P}(\mathbb{R})$ ), it is not clear whether a "nice" (e.g., Hilbert) large vector space could be defined. 
Let us recall when the spaces mentioned above were invented. The latest one is $B_{2}$ invented by Besicovitch in 1926 (see [5]); a year earlier is $\mathscr{A} \mathscr{P}\left(\mathbb{R}^{+}\right)$invented by Bohr [8$10] ; L_{2}(\mathbb{R})$ was invented even earlier. We remark that the function set studied by Wiener mentioned above is not closed under addition either. Some generalizations of $\mathscr{A} \mathscr{P}(\mathbb{R})$, for example, the functions studied in $[1,2,17,21,32-34,38]$, are vector spaces. They are larger than $\mathscr{A} \mathscr{P}(\mathbb{R})$ in $\mathscr{C}(\mathbb{R})$. However, they are the same with $\mathscr{A} \mathscr{P}(\mathbb{R})$ in $H_{2}$. We remark that though $\mathrm{H}_{2}$ is not linear, there have been Banach spaces containing $H_{2}$, for example, the space $B^{2}$ proposed in [11] (in [28] for the discrete setting). However, $[11,28]$ use $\varlimsup$ im instead of lim in (1.1) to construct the spaces. In many cases, lim is needed too. The background of [29] and related problems being pointed out by some authors (e.g., $[27,28,30]$ and references therein) show real needs for new, larger, nice spaces in $H_{2}$.

The purpose of the paper is to propose such spaces. One will see that the new spaces are so natural that they come from what we call generalized trigonometric polynomials in the same way as $\mathscr{A P}(\mathbb{R})$ and $B_{2}$ come from trigonometric polynomials. One will also see that they are so huge that to compare $\mathscr{A} \mathscr{P}(\mathbb{R})$ and $B_{2}$ with them is the same as to compare one point with $\mathbb{R}^{+}$.

The layout of the paper is as follows. In the next section, we show the existence of a larger orthonormal basis. In Section 3, we develop a theory of uniform limit power functions in a way parallel to that of $\mathscr{A P}(\mathbb{R})$ (e.g., $[12,13])$. In Section 4 , we discuss the limit power type functions.

\section{Orthonormal basis}

It is well known that $\left\{e^{i \lambda t}\right\}$ is a complete orthonormal basis in $B_{2}$ [5]. In this section, we consider the set

$$
\left\{e^{i \lambda t^{\alpha}}\right\}
$$

where $\lambda \in \mathbb{R}$ and $0<\alpha<\infty$.

When $\alpha>1$, in radar and sonar terminology, the function $e^{i \lambda t^{\alpha}}$ represents a chirp signal because it is reasonably well defined but steadily rising frequency. By analyzing $f(t)=$ $\sin \left(\pi t^{2}\right)$, [25, Chapter 2] points out the fact that a chirp has a well-defined instantaneous frequency and ordinary Fourier analysis hides the fact. By using Windowed Fourier transform, the signal is reasonably well localized both in time and in frequency. In particular, when $\alpha=2$, the function $e^{i t^{2}}$, being an underlying kernel (e.g., in oscillatory integrals, optics, etc.), has important applications; we refer the reader to [3, 6, 20, 22, 23, 31, 35] for details.

When $\alpha<1$, the function $e^{i \lambda t^{\alpha}}$ behaves conversely.

As $\left\{e^{i \lambda t}\right\}$, the set $\left\{e^{i \lambda t^{\alpha}}\right\}$ is also orthonormal. We show this in the next two theorems.

Theorem 2.1. For $\alpha \geq \beta \geq 0$ with $\alpha \geq 1$ and $\lambda, \mu \in \mathbb{R}$ with $\lambda \neq 0$, the following limit

$$
\lim _{T \rightarrow \infty} \frac{1}{T} \int_{a}^{T+a} e^{i\left(\lambda t^{\alpha}+\mu t^{\beta}\right)} d t= \begin{cases}1, & \alpha=\beta, \mu=-\lambda, \\ 0, & \text { otherwise }\end{cases}
$$

exists uniformly with respect to $a \in \mathbb{R}^{+}$. 
Proof. The conclusion for the case of $\alpha=\beta, \mu=-\lambda$ is obvious, so we only consider the other cases. In these cases, if we can find $a_{0}>0$ such that

$$
\frac{1}{T} \int_{a}^{T+a} e^{i\left(\lambda t^{\alpha}+\mu t^{\beta}\right)} d t \longrightarrow 0 \quad(T \longrightarrow \infty)
$$

uniformly with respect to $a \in\left[a_{0}, \infty\right)$, then we have

$$
\frac{1}{T} \int_{a}^{T+a} e^{i\left(\lambda t^{\alpha}+\mu t^{\beta}\right)} d t \longrightarrow 0 \quad(T \longrightarrow \infty)
$$

uniformly with respect to $a \in \mathbb{R}^{+}$. In fact, for $a \in\left[0, a_{0}\right]$, one has

$$
\begin{aligned}
\left|\frac{1}{T} \int_{a}^{T+a} e^{i\left(\lambda t^{\alpha}+\mu t^{\beta}\right)} d t\right| & \leq \frac{1}{T}\left|\left(\int_{a}^{a_{0}}+\int_{a_{0}}^{T+a_{0}}-\int_{T+a}^{T+a_{0}}\right) e^{i\left(\lambda t^{\alpha}+\mu t^{\beta}\right)} d t\right| \\
& \leq \frac{2 a_{0}}{T}+\frac{1}{T}\left|\int_{a_{0}}^{T+a_{0}} e^{i\left(\lambda t^{\alpha}+\mu t^{\beta}\right)} d t\right| \longrightarrow 0 \quad(T \longrightarrow \infty) .
\end{aligned}
$$

Note that $\alpha, \beta, \lambda$, and $\mu$ are fixed, we can chose $a_{0}>1$ such that $\left|\lambda \alpha+\mu \beta a^{\beta-\alpha}\right| \geq \varepsilon_{0}>0$ for some $\epsilon_{0}>0$ and all $a \in\left[a_{0}, \infty\right)$. In fact, if $\beta=\alpha$ then $\lambda+\mu \neq 0$ and for all $a \in[1, \infty)$ one has $\left|\lambda \alpha+\mu \beta a^{\beta-\alpha}\right|=|\alpha(\lambda+\mu)|=\varepsilon_{0}>0$; if $\beta<\alpha$, then $t^{\beta-\alpha} \rightarrow 0$ as $t \rightarrow \infty$, and therefore there exists $a_{0}>1$ such that for all $a \in\left[a_{0}, \infty\right)$ one has $\left|\lambda \alpha+\mu \beta a^{\beta-\alpha}\right| \geq|\lambda \alpha / 2|=\varepsilon_{0}>0$. For such $a$ and $t \geq a, \lambda \alpha t^{\alpha-1}+\mu \beta t^{\beta-1}=t^{\alpha-1}\left[\lambda \alpha+\mu \beta a^{\beta-\alpha}\right] \neq 0$ and

$$
\begin{aligned}
\int_{a}^{T+a} & e^{i\left(\lambda t^{\alpha}+\mu t^{\beta}\right)} d t \\
& =\int_{a}^{T+a} \frac{e^{i\left(\lambda t^{\alpha}+\mu t^{\beta}\right)} i\left(\lambda \alpha t^{\alpha-1}+\mu \beta t^{\beta-1}\right)}{i\left(\lambda \alpha t^{\alpha-1}+\mu \beta t^{\beta-1}\right)} d t=\int_{a}^{T+a} \frac{e^{i\left(\lambda t^{\alpha}+\mu t^{\beta}\right)} d i\left(\lambda t^{\alpha}+\mu t^{\beta}\right)}{i\left(\lambda \alpha t^{\alpha-1}+\mu \beta t^{\beta-1}\right)} \\
& =\left.\frac{e^{i\left(\lambda t^{\alpha}+\mu t^{\beta}\right)}}{i\left(\lambda \alpha t^{\alpha-1}+\mu \beta t^{\beta-1}\right)}\right|_{a} ^{T+a}-\frac{1}{i} \int_{a}^{T+a} e^{i\left(\lambda t^{\alpha}+\mu t^{\beta}\right)} d \frac{1}{\left(\lambda \alpha t^{\alpha-1}+\mu \beta t^{\beta-1}\right)}=I_{1}+I_{2} .
\end{aligned}
$$

So

$$
\begin{aligned}
\left|I_{1}\right| & \leq \frac{1}{\left|\lambda \alpha(T+a)^{\alpha-1}+\mu \beta(T+a)^{\beta-1}\right|}+\frac{1}{\left|\lambda \alpha a^{\alpha-1}+\mu \beta a^{\beta-1}\right|} \\
& =\frac{1}{(T+a)^{\alpha-1}\left|\lambda \alpha+\mu \beta(T+a)^{\beta-\alpha}\right|}+\frac{1}{a^{\alpha-1}\left|\lambda \alpha+\mu \beta a^{\beta-\alpha}\right|} \leq M_{1},
\end{aligned}
$$

where $M_{1}$ is a constant which is independent of $T$ and $a \in\left[a_{0}, \infty\right)$. 
4 Uniform limit power-type function spaces

To estimate $I_{2}$, we have

$$
\begin{aligned}
\left|I_{2}\right| & \leq \int_{a}^{T+a}\left|\frac{\lambda \alpha(\alpha-1) t^{\alpha-2}+\mu \beta(\beta-1) t^{\beta-2}}{\left(\lambda \alpha t^{\alpha-1}+\mu \beta t^{\beta-1}\right)^{2}}\right| d t=\int_{a}^{T+a}\left|\frac{\lambda \alpha(\alpha-1)+\mu \beta(\beta-1) t^{\beta-\alpha}}{t^{2-\alpha}\left(\lambda \alpha t^{\alpha-1}+\mu \beta t^{\beta-1}\right)^{2}}\right| d t \\
& \leq \int_{a}^{T+a} \frac{|\lambda \alpha(\alpha-1)|+|\mu \beta(\beta-1)|}{t^{\alpha}\left(\lambda \alpha+\mu \beta t^{\beta-\alpha}\right)^{2}} d t \leq M_{2} \int_{a}^{T+a} \frac{1}{t^{\alpha}} d t \\
& =\left\{\begin{array}{l}
M_{2}\left(\ln \left(\frac{T}{a}+1\right)\right) \leq M_{2} \ln (T+1), \quad \alpha=1, \\
M_{2}\left[\left(\frac{1}{a}\right)^{\alpha-1}-\left(\frac{1}{(T+a)}\right)^{\alpha-1}\right], \quad \alpha>1,
\end{array}\right.
\end{aligned}
$$

where $M_{2}$ is a constant which is independent of $T$ and $a \in\left[a_{0}, \infty\right)$.

It follows from (2.6)-(2.8) that

$$
\lim _{T \rightarrow \infty} \frac{1}{T} \int_{a}^{T+a} e^{i\left(\lambda t^{\alpha}+\mu t^{\beta}\right)}=0
$$

uniformly with respect to $a \in\left[a_{0}, \infty\right)$, and therefore with respect to $a \in \mathbb{R}^{+}$, the proof is complete.

Corollary 2.2. For $\alpha \geq 1$ and $\lambda \neq 0$, the limit

$$
\lim _{T \rightarrow \infty} \frac{1}{T} \int_{a}^{T+a} e^{i \lambda t^{\alpha}} d t=0
$$

exists uniformly with respect to $a \in \mathbb{R}^{+}$.

Proof. Put $\mu=0$ in Theorem 2.1 to get the conclusion.

Theorem 2.3. Let $1>\alpha \geq \beta \geq 0$ with $\alpha>0$ and $\lambda, \mu \in \mathbb{R}$ with $\lambda \neq 0$. Then

$$
\lim _{T \rightarrow \infty} \frac{1}{T} \int_{0}^{T} e^{i\left(\lambda t^{\alpha}+\mu t^{\beta}\right)} d t= \begin{cases}1, & \alpha=\beta, \mu=-\lambda \\ 0, & \text { otherwise. }\end{cases}
$$

Proof. First, we consider the case $\alpha=\beta$ and $w=\lambda+\mu \neq 0$,

$$
\int_{1}^{T} e^{i w t^{\alpha}} d t=\int_{1}^{T} \frac{e^{i w t^{\alpha}} i w \alpha t^{\alpha-1}}{i w \alpha t^{\alpha-1}} d t=\left.\frac{e^{i w t^{\alpha}}}{i w \alpha t^{\alpha-1}}\right|_{1} ^{T}-\int_{1}^{T} \frac{e^{i w t^{\alpha}}}{i w \alpha t^{\alpha}}(1-\alpha) d t .
$$


So

$$
\frac{1}{T} \int_{0}^{T} e^{i w t^{\alpha}} d t=\frac{1}{T}\left(\int_{0}^{1}+\int_{1}^{T} e^{i w t^{\alpha}} d t\right) \longrightarrow 0
$$

as $T \rightarrow \infty$.

If $\alpha>\beta$, let $T>a>1$ be so large that $|\lambda \alpha|>\left|\mu \beta a^{\beta-\alpha}\right|$. As in the proof of Theorem 2.1, one has

$$
\begin{gathered}
\int_{a}^{T} e^{i\left(\lambda t^{\alpha}+\mu t^{\beta}\right)} d t=I_{3}+I_{4}, \\
\left|I_{3}\right| \leq \frac{1}{\left(\lambda \alpha T^{\alpha-1}+\mu \beta T^{\beta-1}\right)}+\frac{1}{\left(\lambda \alpha a^{\alpha-1}+\mu \beta a^{\beta-1}\right)} \\
\leq \frac{T^{1-\alpha}}{\left(\lambda \alpha+\mu \beta T^{\beta-\alpha}\right)}+\frac{a^{1-\alpha}}{\left(\lambda \alpha+\mu \beta a^{\beta-\alpha}\right)} \\
\leq T^{1-\alpha}\left[\frac{1}{\left(\lambda \alpha+\mu \beta T^{\beta-\alpha}\right)}+\frac{1}{\left(\lambda \alpha+\mu \beta a^{\beta-\alpha}\right)}\right] \\
\leq T^{1-\alpha}\left[\frac{1}{|\lambda \alpha|-\left|\mu \beta a^{\beta-\alpha}\right|}+\frac{1}{|\lambda \alpha|-\left|\mu \beta a^{\beta-\alpha}\right|}\right] \\
\leq M_{3} T^{1-\alpha} .
\end{gathered}
$$

To estimate $I_{4}$ we have the following:

$$
\begin{aligned}
\left|I_{4}\right| & \leq \int_{a}^{T}\left|\frac{\lambda \alpha(\alpha-1) t^{\alpha-2}+\mu \beta(\beta-1) t^{\beta-2}}{\left(\lambda \alpha t^{\alpha-1}+\mu \beta t^{\beta-1}\right)^{2}}\right| d t \\
& =\int_{a}^{T}\left|\frac{\lambda \alpha(\alpha-1) t^{\alpha-2}+\mu \beta(\beta-1) t^{\beta-2}}{t^{2(\alpha-1)}\left(\lambda \alpha+\mu \beta t^{\beta-\alpha}\right)^{2}}\right| d t \\
& =\int_{a}^{T}\left|\frac{\lambda \alpha(\alpha-1) t^{-\alpha}+\mu \beta(\beta-1) t^{\beta-2 \alpha}}{\left(\lambda \alpha+\mu \beta t^{\beta-\alpha}\right)^{2}}\right| d t \\
& \leq \int_{a}^{T} \frac{|\lambda \alpha(\alpha-1)| t^{-\alpha}+|\mu \beta(\beta-1)| t^{\beta-2 \alpha}}{\left[|\lambda \alpha|-\left|\mu a^{\beta-\alpha}\right|\right]^{2}} d t \\
& \leq\left. M_{4} t^{1-\alpha}\right|_{a} ^{T}+\left.M_{5} t^{1+\beta-2 \alpha}\right|_{a} ^{T},
\end{aligned}
$$

where $M_{4}$ and $M_{5}$ are constants which are independent of $T$.

It follows that

$$
\begin{aligned}
\left|\frac{1}{T} \int_{0}^{T} e^{i\left(\lambda t^{\alpha}+\mu t^{\beta}\right)} d t\right| & =\frac{1}{T}\left|\int_{0}^{a}+\int_{a}^{T} e^{i\left(\lambda t^{\alpha}+\mu t^{\beta}\right)} d t\right| \\
& \leq\left|\frac{1}{T} \int_{0}^{a} e^{i\left(\lambda t^{\alpha}+\mu t^{\beta}\right)} d t\right|+\left|\frac{1}{T} \int_{a}^{T} e^{i\left(\lambda t^{\alpha}+\mu t^{\beta}\right)} d t\right| \longrightarrow 0
\end{aligned}
$$

as $T \rightarrow \infty$. The proof is complete. 
It follows from Theorems 2.1 and 2.3 that

$$
\lim _{T \rightarrow \infty} \frac{1}{T} \int_{0}^{T}\left(e^{i \lambda t^{\alpha}} \cdot e^{-i \mu t^{\beta}}\right) d t= \begin{cases}1, & \alpha=\beta, \mu=\lambda \\ 0, & \text { otherwise. }\end{cases}
$$

That is, the set $\left\{e^{i \lambda t^{\alpha}}\right\}$ constitutes an orthonormal basis.

Remark 2.4. Since the domain of the function $e^{i \lambda t^{\alpha}}$ in general is $\mathbb{R}^{+}$, we consider $\mathbb{R}^{+}$only in the paper. For special numbers of $\alpha$, for example, $\alpha^{\prime} s$ are positive integers, the domain will be $\mathbb{R}$. In this case all the results will hold for $\mathbb{R}$.

\section{Uniform limit power functions}

We call the functions

$$
\sum_{k=1}^{n} a_{k} e^{i \lambda_{k} t^{\alpha}}
$$

$\alpha$-trigonometric polynomial, where $a_{k} \in C$ and $\lambda_{k} \in \mathbb{R}$. As $\mathscr{A} \mathscr{P}(\mathbb{R})$, we have the following definition.

Definition 3.1. Let $\alpha>0$ be fixed. A function $f$ on $\mathbb{R}^{+}$is called uniform limit power if for each $\epsilon>0$ there exists an $\alpha$-trigonometric polynomial $P_{\epsilon}$ such that

$$
\left\|f-P_{\epsilon}\right\|=\sup \left\{\left|f(t)-P_{\epsilon}(t)\right|: t \in \mathbb{R}^{+}\right\}<\epsilon .
$$

Denote by $\mathcal{U} \mathscr{L} \mathscr{P}_{\alpha}\left(\mathbb{R}^{+}\right)$the set of all such functions.

One sees that when $\alpha=1, \mathcal{u} \mathscr{L} \mathscr{P}_{\alpha}\left(\mathbb{R}^{+}\right)=\mathscr{A} \mathscr{P}\left(\mathbb{R}^{+}\right)$. Also one sees that $\mathcal{U} \mathscr{L} \mathscr{P}_{\alpha}\left(\mathbb{R}^{+}\right)$is the completion of $\alpha$-trigonometric polynomial in $C\left(\mathbb{R}^{+}\right)$. Since the set of $\alpha$-trigonometric polynomials are closed under addition, multiplication, and conjugation, so is the completion $\mathscr{U} \mathscr{L} \mathscr{P}_{\alpha}\left(\mathbb{R}^{+}\right)$. Thus we have shown the following statement: $\mathscr{U} \mathscr{L} \mathscr{P}_{\alpha}\left(\mathbb{R}^{+}\right)$is a $C^{*}$ subalgebra of $C\left(\mathbb{R}^{+}\right)$containing the constant functions.

Next, we discuss the Fourier expansion of $f \in \mathscr{U} \mathscr{L} \mathscr{P}_{\alpha}\left(\mathbb{R}^{+}\right)$. First of all, we show that the mean exists.

Theorem 3.2. If $f \in \mathcal{U} \mathscr{L} \mathscr{P}_{\alpha}\left(\mathbb{R}^{+}\right)$, then

$$
\lim _{T \rightarrow \infty} \frac{1}{T} \int_{0}^{T} f(t) d t
$$

exists. In the case of $\alpha \geq 1$,

$$
\lim _{T \rightarrow \infty} \frac{1}{T} \int_{a}^{T+a} f(t) d t
$$

exists uniformly with respect to $a \in \mathbb{R}^{+}$. 
Proof. We first show the theorem in the case that $f$ is an $\alpha$-trigonometric polynomial. Let

$$
f(t)=P(t)=c_{0}+\sum_{k=1}^{n} c_{k} e^{i \lambda_{k} t^{\alpha}} .
$$

Then by Theorems 2.1 and 2.3,

$$
\lim _{T \rightarrow \infty} \frac{1}{T} \int_{0}^{T} P(t) d t=c_{0}
$$

If $f$ is an arbitrary function in $\mathscr{U} \mathscr{L} \mathscr{P}_{\alpha}\left(\mathbb{R}^{+}\right)$then for $\epsilon>0$ there exists an $\alpha$-trigonometric polynomial $P_{\epsilon}$ such that (3.2) holds. Since $\lim _{T \rightarrow \infty}(1 / T) \int_{0}^{T} P_{\epsilon}(t) d t$ exists, we can find a number $T_{0}$ such that when $T_{1}, T_{2}>T_{0}$,

$$
\left|\frac{1}{T_{1}} \int_{0}^{T_{1}} P_{\epsilon}(t) d t-\frac{1}{T_{2}} \int_{0}^{T_{2}} P_{\epsilon}(t) d t\right|<\epsilon .
$$

It follows from (3.2) and the last inequality above that when $T_{1}, T_{2}>T_{0}$,

$$
\begin{aligned}
\left|\frac{1}{T_{1}} \int_{0}^{T_{1}} f(t) d t-\frac{1}{T_{2}} \int_{0}^{T_{2}} f(t) d t\right| \leq & \frac{1}{T_{1}} \int_{0}^{T_{1}}\left|f(t)-P_{\epsilon}(t)\right| d t \\
& +\left|\frac{1}{T_{1}} \int_{0}^{T_{1}} P_{\epsilon}(t) d t-\frac{1}{T_{2}} \int_{0}^{T_{2}} P_{\epsilon}(t) d t\right| \\
& +\frac{1}{T_{2}} \int_{0}^{T_{2}}\left|f(t)-P_{\epsilon}(t)\right| d t<3 \epsilon .
\end{aligned}
$$

Similarly, one shows the existence of the second limit. The proof is complete.

We call the limit in Theorem 3.2 the mean of $f$ and denote it by $M(f)$.

For $\lambda \in \mathbb{R}$ and $f \in \mathscr{U} \mathscr{L} \mathscr{P}_{\alpha}\left(\mathbb{R}^{+}\right)$since the function $f e^{-i \lambda t^{\alpha}}$ is in $\mathcal{U} \mathscr{L} \mathscr{P}_{\alpha}\left(\mathbb{R}^{+}\right)$, the mean exists for the function. We write

$$
a(\lambda)=M\left(f e^{-i \lambda t^{\alpha}}\right)
$$

As the proof for $\mathscr{A} \mathscr{P}\left(\mathbb{R}^{+}\right)$(see $\left.[12,13,18,26,37,38]\right)$, for a function $f \in \mathcal{U} \mathscr{L} \mathscr{P}_{\alpha}\left(\mathbb{R}^{+}\right)$the frequency set

$$
\operatorname{Freq}(f)=\{\lambda \in \mathbb{R}: a(\lambda) \neq 0\}
$$

is countable (or finite). Let $\operatorname{Freq}(f)=\left\{\lambda_{k}\right\}$ and $A_{k}=a\left(\lambda_{k}\right)$. Thus $f$ has an associated Fourier series

$$
f(t) \sim \sum_{k=1}^{\infty} A_{k} e^{i \lambda_{k} t^{\alpha}}
$$

and Parseval's equality holds:

$$
\sum_{k=1}^{\infty}\left|a\left(\lambda_{k}\right)\right|^{2}=M\left(|f|^{2}\right) .
$$


The unique theorem for almost periodic function is well known. That is, distinct almost periodic functions have distinct Fourier series. We point out that this is also true for $u \mathscr{L} \mathscr{P}_{\alpha}\left(\mathbb{R}^{+}\right)$. To show this we need to set up some correspondence between $\mathscr{U} \mathscr{L} \mathscr{P}_{\alpha}\left(\mathbb{R}^{+}\right)$ and $\mathscr{A} \mathscr{P}\left(\mathbb{R}^{+}\right)$. For the $\alpha$-trigonometric polynomial $P_{\epsilon}$ in Definition 3.1, let $s=t^{\alpha}$. Then $P_{\epsilon}$ becomes trigonometric polynomial of $s$. That is,

$$
P_{\epsilon}(s)=\sum_{k=1}^{n} a_{k} e^{i \lambda_{k} s}
$$

For $f \in \mathscr{U} \mathscr{L} \mathscr{P}_{\alpha}\left(\mathbb{R}^{+}\right)$define the function

$$
\tilde{f}(s)=f\left(s^{1 / \alpha}\right) .
$$

Thus, (3.2) becomes

$$
\left|\tilde{f}(s)-P_{\epsilon}(s)\right|<\epsilon \quad\left(s \in \mathbb{R}^{+}\right)
$$

So, $\tilde{f} \in \mathscr{A} \mathscr{P}\left(\mathbb{R}^{+}\right)$. Conversely, let $h \in \mathscr{A} \mathscr{P}\left(\mathbb{R}^{+}\right)$. By the approximation theorem of $\mathscr{A} \mathscr{P}\left(\mathbb{R}^{+}\right)$for $\epsilon>0$, there exists a trigonometric polynomial $\sum_{k=1}^{n} a_{k} e^{i \lambda_{k} s}$ such that

$$
\left|h(s)-\sum_{k=1}^{n} a_{k} e^{i \lambda_{k} s}\right|<\epsilon \quad\left(s \in \mathbb{R}^{+}\right) .
$$

Let $s=t^{\alpha}\left(t \in \mathbb{R}^{+}\right)$and let $\bar{h}(t)=h\left(t^{\alpha}\right)$. It follows that

$$
\left|\bar{h}(t)-\sum_{k=1}^{n} a_{k} e^{i \lambda_{k} t^{\alpha}}\right|<\epsilon \quad\left(t \in \mathbb{R}^{+}\right) .
$$

Therefore, we have $\bar{h} \in \mathcal{U L \mathscr { P }} \alpha\left(\mathbb{R}^{+}\right)$. Thus (3.14) is the correspondence between uLP $\mathscr{P}_{\alpha}\left(\mathbb{R}^{+}\right)$and $\mathscr{A} \mathscr{P}\left(\mathbb{R}^{+}\right)$.

Note the translate property of almost periodic function, that is, for $\epsilon>0$ there exists $l>0$ with the property that any interval $I \subset \mathbb{R}^{+}$of length $l$ has a number $\tau \in I$ such that

$$
|\tilde{f}(s+\tau)-\tilde{f}(s)|<\epsilon \quad\left(s \in \mathbb{R}^{+}\right)
$$

By the correspondence (3.14), we have in fact already shown the following theorem.

Theorem 3.3. Let $f \in C\left(\mathbb{R}^{+}\right)$. Then the following statements are equivalent:

(1) $f \in \mathcal{L} \mathscr{L} \mathscr{P}_{\alpha}\left(\mathbb{R}^{+}\right)$;

(2) for $\epsilon>0$ there exists $l>0$ with the property that any interval $I \subset \mathbb{R}^{+}$of length $l$ has $a$ number $\tau \in I$ such that

$$
\left|f\left[(t+\tau)^{1 / \alpha}\right]-f\left(t^{1 / \alpha}\right)\right|<\epsilon .
$$

Furthermore, if $f \in \mathscr{U} \mathscr{L} \mathscr{P}_{\alpha}\left(\mathbb{R}^{+}\right)$then so is $|f(\cdot)|$.

Now, we make use of the unique theorem for $\tilde{f}$ to get the same conclusion for $f$. 
Lemma 3.4. Let $f \in \mathscr{U} \mathscr{L} \mathscr{P}_{\alpha}\left(\mathbb{R}^{+}\right)$be nonnegative and $f\left(t_{0}\right)>0$ for some $t_{0} \in \mathbb{R}^{+}$. Then $M(f)>0$.

Proof. Let $\tilde{f}$ be the function in (3.14). So $\tilde{f}(s) \geq 0$ and $\tilde{f}\left(s_{0}\right)>0$, where $s_{o}=t_{0}^{\alpha}$. Since $\tilde{f} \in \mathscr{A} \mathscr{P}\left(\mathbb{R}^{+}\right)$, one has

$$
\lim _{T \rightarrow \infty} \frac{1}{T} \int_{a}^{T+a} \tilde{f}(s) d s=M(\tilde{f})>0
$$

uniformly with respect to $a \in \mathbb{R}^{+}$.

To show the theorem we need to discuss two cases.

(1) $\alpha \geq 1$ :

$$
\begin{aligned}
\frac{1}{T} \int_{1}^{T} f(t) d t & =\frac{1}{T} \int_{1}^{T^{\alpha}} f\left(s^{1 / \alpha}\right) \frac{s^{1 / \alpha-1}}{\alpha} d s=\frac{1}{\alpha} \frac{1}{T} \int_{1}^{T^{\alpha}} \frac{\tilde{f}(s)}{s^{1-1 / \alpha}} d s \\
& \geq \frac{1}{\alpha} \frac{1}{T} \int_{1}^{T^{\alpha}} \frac{\tilde{f}(s)}{\left(T^{\alpha}\right)^{1-1 / \alpha}} d s=\frac{1}{\alpha} \frac{1}{T^{\alpha}} \int_{1}^{T^{\alpha}} \tilde{f}(s) d s
\end{aligned}
$$

It follows from (3.20) that

$$
M(f(t)) \geq \frac{1}{\alpha} M(\tilde{f}(s))>0 .
$$

(2) $0<\alpha<1$ : in this case,

$$
\begin{aligned}
\frac{1}{T} \int_{0}^{T} f(t) d t & =\frac{1}{T} \int_{0}^{T^{\alpha}} f\left(s^{1 / \alpha}\right) \frac{s^{1 / \alpha-1}}{\alpha} d s=\frac{1}{\alpha} \frac{1}{T} \int_{0}^{T^{\alpha}} \tilde{f}(s) s^{1 / \alpha-1} d s \\
& \geq \frac{1}{\alpha} \frac{1}{T} \int_{T^{\alpha / 2}}^{T^{\alpha}} \tilde{f}(s)\left(\frac{T^{\alpha}}{2}\right)^{1 / \alpha-1} d s=\frac{1}{\alpha} \frac{1}{T} \frac{T^{1-\alpha}}{2^{1 / \alpha-1}} \int_{T^{\alpha} / 2}^{T^{\alpha}} \tilde{f}(s) d s \\
& =\frac{1}{\alpha 2^{1 / \alpha}} \frac{1}{T^{\alpha / 2}} \int_{T^{\alpha / 2}}^{T^{\alpha}} \tilde{f}(s) d s .
\end{aligned}
$$

So, in this case we also have

$$
M(f(t)) \geq \frac{1}{\alpha 2^{1 / \alpha}} M(\tilde{f}(s))>0
$$

The proof is complete.

By the lemma above we are able to show the following unique theorem.

Theorem 3.5. Distinct uniform limit power functions have distinct Fourier series.

Proof. Suppose that the distinct functions $f_{1}, f_{2} \in \mathcal{U} \mathscr{L} \mathscr{P}_{\alpha}\left(\mathbb{R}^{+}\right)$have the same Fourier series. Then $f_{1}-f_{2}$ will have Fourier series of all term zero. By Parseval's equality $M\left(\mid f_{1}-\right.$ $\left.\left.f_{2}\right|^{2}\right)=0$. However, by Lemma 3.4 we get $M\left(\left|f_{1}-f_{2}\right|^{2}\right)>0$. This is a contraction. The proof is complete. 
For $f(t) \in U \mathscr{L} \mathscr{P}_{\alpha}\left(\mathbb{R}^{+}\right)$since $\tilde{f}(s) \in \mathscr{A} \mathscr{P}\left(\mathbb{R}^{+}\right)$, the function $\tilde{f}$ has an associated Fourier series

$$
\tilde{f}(s) \sim \sum_{k=1}^{\infty} a_{k} e^{i \mu_{k} s}
$$

where $a_{k}=M\left(\tilde{f}(s) e^{-i \mu_{k} s}\right)$. It is well known (e.g., see $\left.[12,13,38]\right)$ that $\tilde{f}$ can be approximated uniformly on $\mathbb{R}$ by the Bocher-Fejér trigonometric polynomials

$$
\sigma_{m}(s)=\sum_{k=1}^{n(m)} r_{m, k} a_{k} e^{i \mu_{k} s}
$$

where the rational numbers $0 \leq r_{m, k} \leq 1$ and $\lim _{m \rightarrow \infty} r_{m, k}=1$. Thus, replacing $s$ in (3.26) by $t^{\alpha}$ we have

$$
\left|\sum_{k=1}^{n(m)} r_{m, k} a_{k} e^{i \mu_{k} t^{\alpha}}-\tilde{f}\left(t^{\alpha}\right)\right|=\left|\sum_{k=1}^{n(m)} r_{m, k} a_{k} e^{i \mu_{k} t^{\alpha}}-f(t)\right| \longrightarrow 0
$$

uniformly on $\mathbb{R}^{+}$.

The following remark tells us an important conclusion.

Remark 3.6. In the section all the results are achieved under the assumption of fixed $\alpha$. We may release the restriction on $\alpha$. Let $\left\{\alpha_{1}, \alpha_{2}, \ldots, \alpha_{n}\right\}$ be nonnegative sequence. A generalized trigonometric polynomial is a function of the form

$$
\sum_{k=1}^{n} a_{k} e^{i \lambda_{k} t^{\alpha_{k}}}
$$

where $a_{k} \in C$ and $\lambda_{k} \in \mathbb{R}, 1 \leq k \leq n$. If in Definition 3.1 $P_{\epsilon}$ is a generalized trigonometric polynomial, then the function $f$ is also called uniform limit power and $U \mathscr{L} \mathscr{P}\left(\mathbb{R}^{+}\right)$is denoted the set of all such functions. It is not difficult to show that $\mathscr{L} \mathscr{L}\left(\mathbb{R}^{+}\right)$is a Banach space and (3.10)-(3.12) are valid. For the question if an $f \in \mathcal{U} \mathscr{P}\left(\mathbb{R}^{+}\right)$can be approximated by the Bochner-Fejer polynomials, as well as how to construct the polynomials, we refer the reader to $[40,41]$ for details. Also

$$
\mathcal{L} \mathscr{P}\left(\mathbb{R}^{+}\right)=\overline{\operatorname{span}}\left\{u \mathscr{L} \mathscr{P}_{\alpha}\left(\mathbb{R}^{+}\right): 0<\alpha<\infty\right\} \subset C\left(\mathbb{R}^{+}\right) \cap H_{2}
$$

where the closure is taken in $C\left(\mathbb{R}^{+}\right)$. One can see how huge $\mathscr{U} \mathscr{L} \mathscr{P}\left(\mathbb{R}^{+}\right)$is by comparing with $\mathscr{A} \mathscr{P}\left(\mathbb{R}^{+}\right)$.

Remark 3.7. As chirps, some existing results enable us to analyze and reconstruct $f \in$ uLSP $\left(\mathbb{R}^{+}\right)$. For example, by [30, Theorem 2.1] a windowed Fourier transform of $f$ exists, by Theorem 2.2 of the same paper the transform satisfies some Parseval's relation, and by Theorem 2.4 of that paper again a generalized frame exists.

One more remark is needed to end the section. 
Remark 3.8. (1) The conclusion in the paragraph before Remark 2.4 is mentioned in [39, Section 2] without proof. Here we not only prove it in details, but we also distinguish the limits between the case $\alpha \geq 1$ and the case $\alpha<1$ in Theorems 2.1 and 2.3, respectively. (2) Also, the results in Section 3 are presented in [39, Section 2] in an abstract-like form. To convince the reader the correctness of these results, we present and prove them in details here.

\section{Limit power type functions}

In this section, we will define and investigate three types of limit power function which are corresponding to the three types of well-known almost periodic functions (e.g., see $[1,2,17,21,31,33,34,38])$.

Let

$$
\begin{array}{r}
C_{0}\left(\mathbb{R}^{+}\right)=\left\{\varphi \in C\left(\mathbb{R}^{+}\right): \lim _{t \rightarrow \infty} \varphi(t)=0\right\}, \\
\mathscr{P} \mathscr{A} \mathscr{P}_{0}\left(\mathbb{R}^{+}\right)=\left\{\varphi \in C\left(\mathbb{R}^{+}\right): M(|\varphi|)=0\right\} .
\end{array}
$$

Definition 4.1. Let $f \in C\left(\mathbb{R}^{+}\right)$. A function $f$ is called asymptotic limit power if

$$
f(t)=g(t)+\varphi(t) \quad\left(t \in \mathbb{R}^{+}\right)
$$

where $g \in \mathscr{U} \mathscr{L} \mathscr{P}_{\alpha}\left(\mathbb{R}^{+}\right)$and $\varphi \in C_{0}\left(\mathbb{R}^{+}\right)$. Denote by $\mathscr{A} \mathscr{L} \mathscr{P}_{\alpha}\left(\mathbb{R}^{+}\right)$all such functions.

By (3.14), we have

$$
\tilde{f}(s)=\tilde{g}(s)+\tilde{\varphi}(s)
$$

It is easy to check that $\varphi(t)$ is in $C_{0}\left(\mathbb{R}^{+}\right)$if and only if $\tilde{\varphi}(s)$ is in $C_{0}\left(\mathbb{R}^{+}\right)$. Since $\tilde{g}(s) \in$ $\mathscr{A} \mathscr{P}\left(\mathbb{R}^{+}\right)$, one gets that $\tilde{f}(s) \in \mathscr{A} \mathscr{A} \mathscr{P}\left(\mathbb{R}^{+}\right)$, the space of asymptotically almost periodic functions. Therefore, (3.14) is also a correspondence between $\mathscr{A} \mathscr{A} \mathscr{P}\left(\mathbb{R}^{+}\right)$and $\mathscr{A} \mathscr{L} \mathscr{P}_{\alpha}\left(\mathbb{R}^{+}\right)$. Note the characterization of $\mathscr{A} \mathscr{A} \mathscr{P}\left(\mathbb{R}^{+}\right)$(e.g., see [38, Theorem 1.2.11]), we have the following corresponding characterization.

THEOREM 4.2. Let $f \in C\left(\mathbb{R}^{+}\right)$. Then the following statements are equivalent:

(1) $\tilde{f} \in \mathscr{A} \mathscr{L} \mathscr{P}_{\alpha}\left(\mathbb{R}^{+}\right)$;

(2) the set $\left\{f\left[(t+x)^{1 / \alpha}\right]: x \in \mathbb{R}^{+}\right\}$is relatively compact in $C\left(\mathbb{R}^{+}\right)$;

(3) for any $\epsilon>0$ there exists a bounded closed interval $C=[0, a]$ and $l>0$ such that any interval $I \subset \mathbb{R}^{+}$of length 1 has a number $\tau \in I$ with the property

$$
\left|f\left[(t+\tau)^{1 / \alpha}\right]-f(t)^{1 / \alpha}\right|<\epsilon \quad\left(t, t+\tau \in \mathbb{R}^{+} \backslash C\right) .
$$

If we only require the set in Theorem 4.2(2) to be weakly compact, then we get the following concept.

Definition 4.3. An $f \in C\left(\mathbb{R}^{+}\right)$is called weak limit power if the set in Theorem 4.2(2) is weakly compact in $C\left(\mathbb{R}^{+}\right)$. Denote by $\mathscr{W} \mathscr{L} \mathscr{P}_{\alpha}\left(\mathbb{R}^{+}\right)$of all such functions. 
To get the decomposition of a function $f \in \mathscr{W} \mathscr{L} \mathscr{P}_{\alpha}\left(\mathbb{R}^{+}\right)$, we introduce the following set:

$$
\mathscr{W} \mathscr{L} \mathscr{P}_{0}(b) \mathbb{R}^{+}=\left\{\varphi \in \mathscr{W} \mathscr{L} \mathscr{P}_{\alpha}\left(\mathbb{R}^{+}\right): 0 \in \overline{\left\{\varphi\left[(t+x)^{1 / \alpha}\right]: x \in \mathbb{R}^{+}\right\}}\right\}
$$

where the closure is taken under weak topology in $C\left(\mathbb{R}^{+}\right)$. The following result is a correspondence in $\mathscr{W} \mathscr{L} \mathscr{P}_{\alpha}\left(\mathbb{R}^{+}\right)$to that in $\mathscr{W} \mathscr{A} \mathscr{P}\left(\mathbb{R}^{+}\right)$.

Theorem 4.4. Let $f \in C\left(\mathbb{R}^{+}\right)$. Then the following statements are equivalent:

(1) $f \in \mathscr{W} \mathscr{L} \mathscr{P}_{\alpha}\left(\mathbb{R}^{+}\right)$;

(2) $f=g+\varphi$, where $g \in \mathcal{U} \mathscr{L} \mathscr{P}_{\alpha}\left(\mathbb{R}^{+}\right)$and $\varphi \in \mathscr{W} \mathscr{L} \mathscr{P}_{0}\left(\mathbb{R}^{+}\right)$.

Finally we give the following concept.

Definition 4.5. An $f \in C\left(\mathbb{R}^{+}\right)$is called pseudolimit power if $f$ has the form $f=g+\varphi$, where $g \in \mathscr{U} \mathscr{L} \mathscr{P}_{\alpha}\left(\mathbb{R}^{+}\right)$and $\varphi \in \mathscr{P} \mathscr{A} \mathscr{P}_{0}\left(\mathbb{R}^{+}\right)$. Denote by $\mathscr{P} \mathscr{L} \mathscr{P}_{\alpha}\left(\mathbb{R}^{+}\right)$all such functions.

Let $f \in \mathscr{P} \mathscr{L} \mathscr{P}_{\alpha}\left(\mathbb{R}^{+}\right)$. Since $f(t) e^{-i \lambda t^{\alpha}}=g(t) e^{-i \lambda t^{\alpha}}+\varphi(t) e^{-i \lambda t^{\alpha}}$ and

$$
\lim _{T \rightarrow \infty} 1 / T \int_{0}^{T} f(t) e^{-i \lambda t^{\alpha}} d t=M\left(g e^{-i \lambda t^{\alpha}}\right)
$$

for all $\lambda \in \mathbb{R}$, Theorem 3.5 implies that $\mathscr{P} \mathscr{L} \mathscr{P}_{\alpha}\left(\mathbb{R}^{+}\right)$is a direct sum of $\mathcal{U} \mathscr{L} \mathscr{P}_{\alpha}\left(\mathbb{R}^{+}\right)$and $\mathscr{P} \mathscr{A} \mathscr{P}_{0}\left(\mathbb{R}^{+}\right)$. Since the ranges $R f$ and $R \tilde{f}$ are the same (so $R g$ and $R \tilde{g}, R \varphi$ and $R \tilde{\varphi}$ ) and $\overline{R \tilde{f}} \supset R \tilde{g}$ [38, Lemma 1.5.2], we have $\overline{R f} \supset R g$. By this, we can show the following theorem.

Theorem 4.6. $\mathscr{P} \mathscr{L} \mathscr{P}_{\alpha}\left(\mathbb{R}^{+}\right)$is a Banach space.

Proof. Let $\left\{f_{n}\right\} \subset \mathscr{P} \mathscr{L} \mathscr{P}_{\alpha}\left(\mathbb{R}^{+}\right)$be Cauchy. Since $\overline{R f_{n}} \supset R g_{n},\left\{g_{n}\right\}$ is Cauchy too. Note $\mathcal{U L} \mathscr{P}_{\alpha}\left(\mathbb{R}^{+}\right)$is closed in $C\left(\mathbb{R}^{+}\right)$, there exists $g \in \mathcal{U} \mathscr{L} \mathscr{P}_{\alpha}\left(\mathbb{R}^{+}\right)$such that $\left\|g_{n}-g\right\| \rightarrow 0$ as $n \rightarrow \infty$. Since $\left\{f_{n}-g_{n}\right\}$ is also Cauchy and $\mathscr{P} \mathscr{A} \mathscr{P}_{0}\left(\mathbb{R}^{+}\right)$is closed in $C\left(\mathbb{R}^{+}\right)$, there exists $\varphi \in \mathscr{P} \mathscr{A} \mathscr{P}_{0}\left(\mathbb{R}^{+}\right)$such that $\left\|\varphi_{n}-\varphi\right\| \rightarrow 0$ as $n \rightarrow \infty$. Let $f=g+\varphi$. Then $f \in \mathscr{P} \mathscr{L} \mathscr{P}_{\alpha}\left(\mathbb{R}^{+}\right)$ and $\left\|f_{n}-f\right\| \rightarrow 0$ as $n \rightarrow \infty$. The proof is complete.

Since

$$
\mathscr{A} \mathscr{P}\left(\mathbb{R}^{+}\right) \subset \mathscr{A} \mathscr{A} \mathscr{P}\left(\mathbb{R}^{+}\right) \subset \mathscr{W} \mathscr{A} \mathscr{P}\left(\mathbb{R}^{+}\right) \subset \mathscr{P} \mathscr{A} \mathscr{P}\left(\mathbb{R}^{+}\right)
$$

one has the following inclusion relationship:

$$
\mathscr{u} \mathscr{L} \mathscr{P}_{\alpha}\left(\mathbb{R}^{+}\right) \subset \mathscr{A} \mathscr{L} \mathscr{P}_{\alpha}\left(\mathbb{R}^{+}\right) \subset \mathscr{W} \mathscr{L} \mathscr{P}_{\alpha}\left(\mathbb{R}^{+}\right) \subset \mathscr{P} \mathscr{L} \mathscr{P}_{\alpha}\left(\mathbb{R}^{+}\right)
$$

\section{Acknowledgments}

The authors would like to thank the referees for the valuable comments. The research is supported by NSF of China (no.10671046). 


\section{References}

[1] A. I. Alonso, J. Hong, and J. Rojo, A class of ergodic solutions of differential equations with piecewise constant arguments, Dynamic Systems and Applications 7 (1998), no. 4, 561-574.

[2] B. Basit and C. Zhang, New almost periodic type functions and solutions of differential equations, Canadian Journal of Mathematics 48 (1996), no. 6, 1138-1153.

[3] J. J. Benedetto, Harmonic Analysis and Applications, Studies in Advanced Mathematics, CRC Press, Florida, 1997.

[4] J. F. Berglund, H. D. Junghenn, and P. Milnes, Analysis on Semigroups: Function Spaces, Compactifications, Representations, Canadian Mathematical Society Series of Monographs and Advanced Texts, John Wiley \& Sons, New York, 1989.

[5] A. S. Besicovitch, Almost Periodic Functions, Dover, New York, 1955.

[6] R. E. Blahut, W. Miller Jr., and C. H. Wilcox, Radar and Sonar. Part I, The IMA Volumes in Mathematics and Its Applications, vol. 32, Springer, New York, 1991.

[7] S. Bochner, A new approach to almost periodicity, Proceedings of the National Academy of Sciences of the United States of America 48 (1962), 2039-2043.

[8] H. Bohr, Zur theorie der fastperiolischen funktionen, Acta Mathematica 45 (1925), no. 1, 19-127.

[9] _ Zur theorie der fastperiolischen funktionen. II, Acta Mathematica 46 (1926), 101-214.

[10] _ Zur theorie der fastperiolischen funktionen. III, Acta Mathematica 47 (1926), 237-281.

[11] H. Bohr and E. Følner, On some types of functional spaces. A contribution to the theory of almost periodic functions, Acta Mathematica 76 (1945), 31-155.

[12] C. Corduneanu, Almost Periodic Functions, 1st ed., John Wiley \& Sons, New York, 1968.

[13]_ Almost Periodic Functions, 2nd ed., Chelsea, New York, 1989.

[14] G. Da Prato and A. Ichikawa, Optimal control of linear systems with almost periodic inputs, SIAM Journal on Control and Optimization 25 (1987), no. 4, 1007-1019.

[15] K. de Leeuw and I. Glicksberg, Applications of almost periodic compactifications, Acta Mathematica 105 (1961), 63-97.

[16] J. C. Doyle, B. A. Francis, and A. R. Tannenbaum, Feedback Control Theory, Macmillan, New York, 1992.

[17] W. F. Eberlein, Abstract ergodic theorems and weak almost periodic functions, Transactions of the American Mathematical Society 67 (1949), no. 1, 217-240.

[18] A. M. Fink, Almost periodic functions invented for specific purposes, SIAM Review 14 (1972), no. 4, $572-581$.

[19] H. Frid, Decay of almost periodic solutions of conservation laws, Archive for Rational Mechanics and Analysis 161 (2002), no. 1, 43-64.

[20] J. W. Goodman, Introduction to Fourier Optics, McGraw-Hill, New York, 1968.

[21] J. Hong and R. Obaya, Ergodic type solutions of some differential equations, Differential Equations and Nonlinear Mechanics, Math. Appl., vol. 528, Kluwer Academic, Dordrecht, 2001, pp. 135152.

[22] L. Hörmander, The Analysis of Linear Partial Differential Operators. I, Fundamental Principles of Mathematical Sciences, vol. 256, Springer, Berlin, 1983.

[23] — The Analysis of Linear Partial Differential Operators. II, Fundamental Principles of Mathematical Sciences, vol. 257, Springer, Berlin, 1983.

[24] B. Jacob, M. Larsen, and H. Zwart, Corrections and extensions of: "Optimal control of linear systems with almost periodic inputs" by G. Da Prato and A. Ichikawa, SIAM Journal on Control and Optimization 36 (1998), no. 4, 1473-1480.

[25] G. Kaiser, A Friendly Guide to Wavelets, Birkhäuser Boston, Massachusetts, 1994.

[26] B. M. Levitan, Almost Periodic Functions, Higher Education Press, Beijing, 1956.

[27] P. M. Mäkilä, On three puzzles in robust control, IEEE Transactions on Automatic Control 45 (2000), no. 3, 552-556. 


\section{Uniform limit power-type function spaces}

[28] P. M. Mäkilä, J. R. Partington, and T. Norlander, Bounded power signal spaces for robust control and modeling, SIAM Journal on Control and Optimization 37 (1999), no. 1, 92-117.

[29] J. Mari, A counterexample in power signals space, IEEE Transactions on Automatic Control 41 (1996), no. 1, 115-116.

[30] J. R. Partington and B. Ünalmış, On the windowed Fourier transform and wavelet transform of almost periodic functions, Applied and Computational Harmonic Analysis 10 (2001), no. 1, 4560.

[31] A. W. Rihaczek, Principle of High-Resolution Radar, Peninsula, California, 1985.

[32] W. Rudin, Weak almost periodic functions and Fourier-Stieltjes transforms, Duke Mathematical Journal 26 (1959), no. 2, 215-220.

[33] W. M. Ruess and W. H. Summers, Ergodic theorems for semigroups of operators, Proceedings of the American Mathematical Society 114 (1992), no. 2, 423-432.

[34] D. Sarason, Toeplitz operators with semi-almost periodic symbols, Duke Mathematical Journal 44 (1977), no. 2, 357-364.

[35] E. M. Stein, Harmonic Analysis: Real-Variable Methods, Orthogonality, and Oscillatory Integrals, Princeton Mathematical Series, vol. 43, Princeton University Press, New Jersey, 1993, with the assistance of T. S. Murphy.

[36] N. Wiener, The Fourier Integral and Certain of Its Applications, Cambridge University Press, Cambridge, 1933.

[37] S. Zaidman, Almost-Periodic Functions in Abstract Spaces, Research Notes in Mathematics, vol. 126, Pitman, Massachusetts, 1985.

[38] C. Zhang, Almost Periodic Type Functions and Ergodicity, Science Press, Beijing, 2003.

[39] _ New limit power function spaces, IEEE Transactions on Automatic Control 49 (2004), no. 5, 763-766.

[40] Strong limit power functions, Journal of Fourier Analysis and Applications 12 (2006), no. 3, 291-307.

[41] C. Zhang and C. Meng, $C^{*}$-algebra of strong limit power functions, IEEE Transactions on Automatic Control 51 (2006), no. 5, 828-831.

[42] K. Zhou, J. C. Doyle, and K. Glover, Robust and Optimal Control, Prentice-Hall, New Jersey, 1996.

Chuanyi Zhang: Department of Mathematics, Harbin Institute of Technology, Harbin 150001, China E-mail address: czhang@hope.hit.edu.cn

Weiguo Liu: Department of Mathematics, Harbin Institute of Technology, Harbin 150001, China E-mail address: czhang@hit.edu.cn 


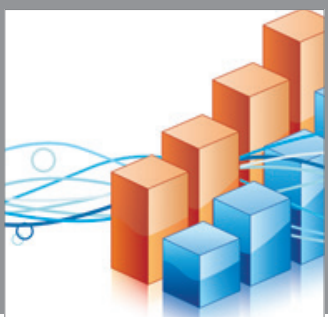

Advances in

Operations Research

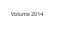

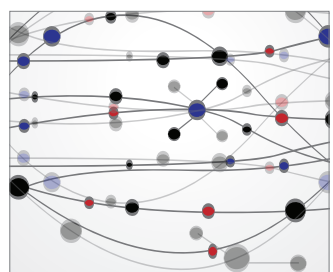

\section{The Scientific} World Journal
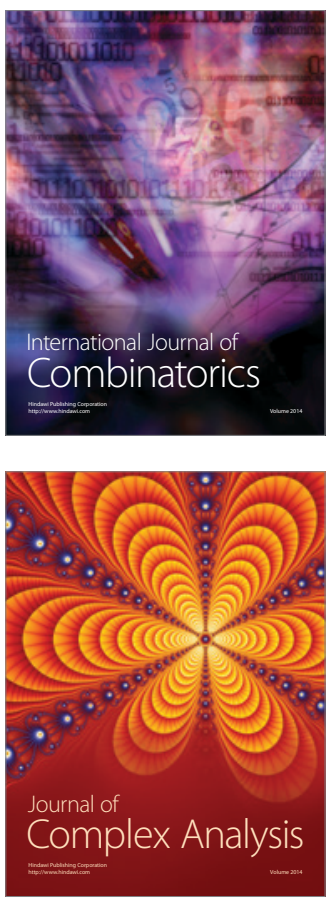

International Journal of

Mathematics and

Mathematical

Sciences
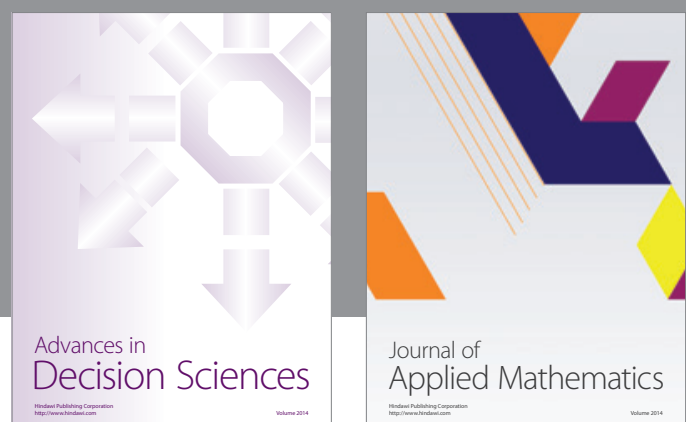

Journal of

Applied Mathematics
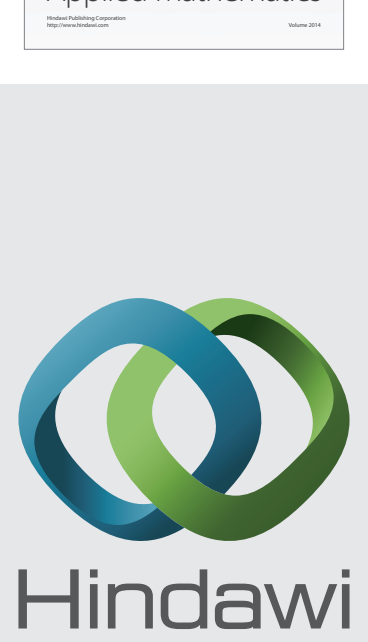

Submit your manuscripts at http://www.hindawi.com
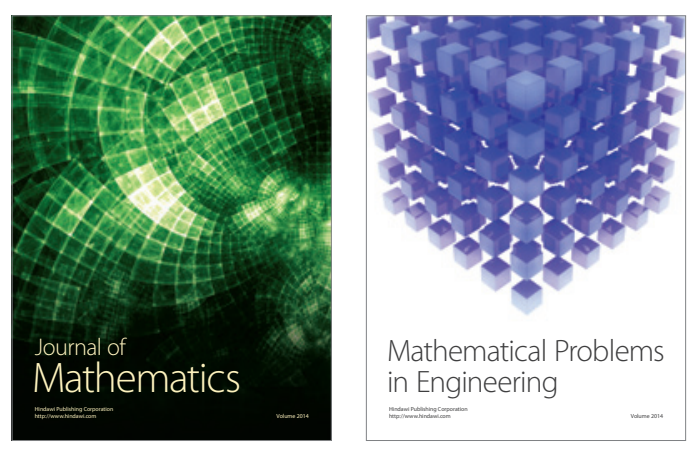

Mathematical Problems in Engineering
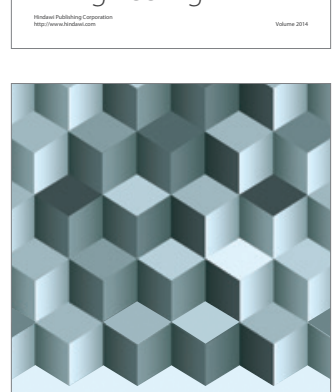

Journal of

Function Spaces
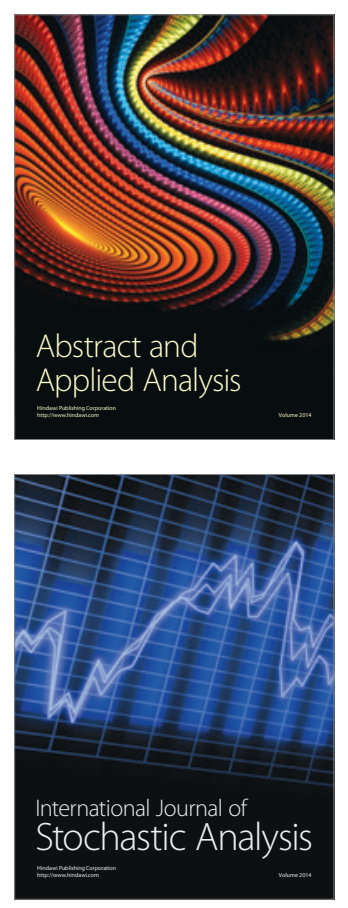

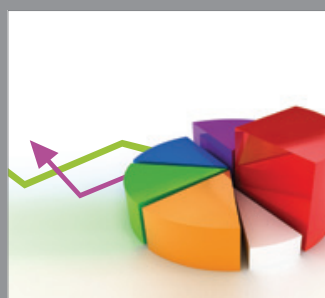

ournal of

Probability and Statistics

Promensencen
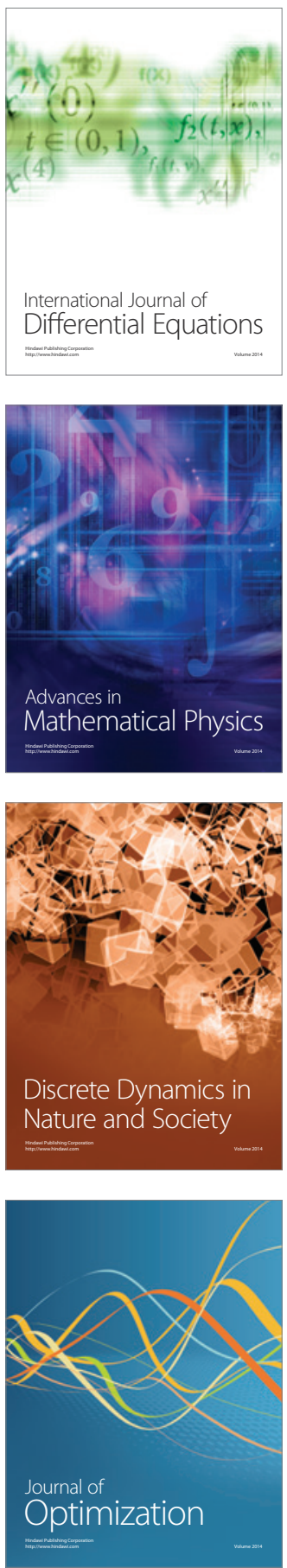